\title{
Denosumab after 8 years
}

\author{
I. R. Reid ${ }^{1,2}$
}

Received: 6 August 2015 / Accepted: 28 September 2015 / Published online: 16 October 2015

(C) International Osteoporosis Foundation and National Osteoporosis Foundation 2015

It is now more than 20 years since the alendronate phase 3 program produced the first trials demonstrating global antifracture efficacy for an osteoporosis medication [1, 2]. This brought the osteoporosis field into the era of evidence-based medicine. The euphoria that followed from knowing that the interventions we were prescribing really were preventing fractures soon passed as durations of therapy reached 3 years, the endpoint of most fracture prevention clinical trials. A series of unanswered questions then arose. How long should treatment be continued? Are there long-term side-effects? Do these drugs continue to prevent fractures and, if so, is their continued use necessary for sustained fracture prevention? For alendronate, some of these questions were answered by the FLEX trial, which suggested that dose reduction with long-term use did not reduce efficacy, and that for patients whose femoral neck bone density was no longer in the osteoporotic range, drug discontinuation did not negatively impact on fracture rate $[3,4]$. Similar data have now been published from the zoledronate extension studies [5].

In the last decade, osteonecrosis of the jaw (ONJ) and transverse stress fractures of the femoral shaft, referred to as an atypical femoral fractures (AFFs), have emerged as significant new safety concerns associated with the long-term use of anti-resorptive drugs. Most information on these problems is from case series or from analyses of medical claims databases.

I. R. Reid

i.reid@auckland.ac.nz

1 Faculty of Medical and Health Sciences, University of Auckland, Private Bag 92019, Auckland, New Zealand

2 Department of Endocrinology, Auckland District Health Board, Auckland, New Zealand
Some such studies have even suggested that the incidence of atypical femoral fractures attributable to bisphosphonate use might exceed the number of hip fractures prevented by these drugs [6], though other analyses do not support this conclusion [7]. In this context, longer-term data from clinical trials become critically important in assessing the ongoing safety and efficacy of medications for osteoporosis. Longer clinical trials are desirable, though maintaining patients at high risk of fracture on placebo raises ethical and practical issues. The next-best option is to continue long-term follow-up of the cohorts of patients involved in the pivotal clinical trials. While not as robust as a longterm randomized controlled trial because a control group is not continued on placebo, this strategy does provide longitudinal data on a well-characterized cohort, allowing fracture rates and adverse events to be related to duration of therapy. It also allows documentation of chronic effects on bone turnover markers and bone density. The loss of a control group for these measures is not such a problem, since current technology usually generates stable measurements, and the evolution of these parameters in placebo-treated women with osteoporosis is already well documented.

The recent report by Papapoulos et al. in Osteoporosis International provides important new information regarding the efficacy and safety of the anti-RANKL monoclonal antibody, denosumab [8]. This drug is a potent antiresorptive which reduces median bone formation rate to zero after 2-3 years use [9], raising concerns regarding over-suppression of bone turnover. In contrast to the bisphosphonates, it is not bound to bone, so its anti-resorptive effects abate rapidly at the end of the 6-month inter-dose interval, possibly allaying these concerns to some extent. These new data from Papapoulos et al demonstrate stable suppression of pre-dose bone turnover markers between 1 and 8 years 
of denosumab use. However, there is a wide range of values of C-telopeptide, indicating that $>50 \%$ of patients have bone resorption rates well within the postmenopausal normal range at the time they are due for re-dosing. This appears to contrast with similar data from alendronate and zoledronate, which seem to produce more consistent suppression of resorption $[3,10]$. Also contrasting with bisphosphonates is the continued increase in bone density with long-term use, albeit not at the same rate observed in the first 1-2 years of treatment. This is not confined to the spine, where increases often represent accumulation of degenerative artifact rather than improvement in the actual density of bone, but is also observed in the hip and forearm. With zoledronate, total hip density peaks after 4.5 years of treatment at $5 \%$ above baseline, compared with $8 \%$ for denosumab at 8 years.

Consistent with the density and markers findings, fracture rates remain low out to 8 years. These findings need to be interpreted with caution, since there is a steady attrition of patients from the study, from whom data are not available. It is probably the frailer members of the cohort who will discontinue follow-up, and these are the individuals with the highest fracture risk. Therefore, these data may be biased towards under-estimating the true fracture rate. Also, the confidence intervals around estimates of fracture rate are wide as a result of small numbers of events, so circumspection in interpretation is necessary. With these caveats, the Papapoulos study does suggest long-term maintenance of reduced fracture rates to 8 years with denosumab, and no concrete evidence that sustained suppression of bone turnover results in a generalized increase in skeletal fragility.

Also recently published in Osteoporosis International is an analysis of a subset of these data from some of the same investigators [11]. This paper re-presents data in Papapoulos, considering the non-vertebral fracture incidence to 7 years ( 3 years of denosumab or placebo in the core trial, plus 4 years of the study extension during which all subjects received denosumab). The authors focus particularly on fracture rates in year 4 of active treatment, concluding that there is a further $36 \%$ reduction in non-vertebral fracture rate from year 3 to year 4 of denosumab use. Since bone turnover has been stably suppressed for 3 years, it seems biologically implausible that a step change in fracture rate would occur at this time. It is instructive to note the fluctuation in the year-byyear fracture rates, and to note the confidence intervals around these estimates. Fracture rates in years 6 and 7 are very similar to those in year 2. It is also instructive to consider the vertebral fracture rates, only presented in the Papapoulos paper, which apparently doubled between year 2 and years $4-5$ of treatment. This is unlikely to be a real effect, as is the change in non-vertebral fracture numbers in the opposite direction over the same period. Both observations highlight the dangers of post hoc analyses not founded on biologically based hypotheses. If vertebral and non-vertebral fractures are summed, then in year 2, 2.8\% of participants had a fracture, and in years 4 and $5,2.9 \%$ fractured, suggesting stable fracture incidence. This is also the finding of the just published study of Gnant, which demonstrated stable anti-fracture efficacy of denosumab over 6 years in women receiving aromatase inhibitor treatment for early stage breast cancer [12].

The safety results at 8 years are particularly important. There were eight patients with ONJ, all diagnosed during the study extension, though three were in the cross-over group. This equates to an incidence of $\sim 4 / 10,000$ patient-years. By comparison, there was one case of ONJ in both the zoledronate and placebo groups in Black et al [13], 1 during 1914 patients-years of observation in the study extensions [10, 14], and no cases in the other phase 3 zoledronate study [15]. These data do not suggest a difference in incidences of this problem with these two medications. There were two AFFs with denosumab, one in each group during the extension, equating to $\sim 1 / 10,000$ patient-years. There were no AFFs in the zoledronate extensions, and a rate of 2.3 subtrochanteric fractures $/ 10,000$ patient-years was found in a combined database of zoledronate and alendronate trials, though whether any of these fractures were atypical is not known [16]. Thus, these sparse data do not establish a difference in AFFs among these commonly used therapeutic options.

In conclusion, continuous use of denosumab to 8 years appears to be safe and to maintain reduced fracture rates. The suggestion of a further abrupt reduction in fracture risk after year 3 seems improbable to this author, and not well supported by the totality of the data available. When targeted to individuals with a high enough fracture risk for its use to be cost-effective, denosumab represents an important addition to our means of combating the mounting problem of osteoporotic fractures in our aging population.

Acknowledgement This paper was supported by the Health Research Council of New Zealand.

Conflict of interest Dr Reid has received grant funding and honoraria from Amgen, Novartis and Merck.

\section{References}

1. Liberman UA, Weiss SR, Broll J et al (1995) Effect of oral alendronate on bone mineral density and the incidence of fractures in postmenopausal osteoporosis. N Engl J Med 333:1437-1443

2. Black DM, Cummings SR, Karpf DB et al (1996) Randomised trial of effect of alendronate on risk of fracture in women with existing vertebral fractures. Lancet 348:1535-1541

3. Black DM, Schwartz AV, Ensrud KE et al (2006) Effects of continuing or stopping alendronate after 5 years of treatment-The Fracture Intervention Trial long-term extension (FLEX): a randomized trial. JAMA 296:2927-2938

4. Schwartz AV, Bauer DC, Cummings SR et al (2010) Efficacy of continued alendronate for fractures in women with and without prevalent vertebral fracture: the FLEX trial. J Bone Miner Res 25:976-982 
5. Cosman F, Cauley JA, Eastell R et al (2014) Reassessment of fracture risk in women after 3 years of treatment with zoledronic acid: when is it reasonable to discontinue treatment? J Clin Endocrinol Metab 99:4546-4554

6. Schilcher J, Koeppen V, Aspenberg P et al (2014) Risk of atypical femoral fracture during and after bisphosphonate use. N Engl J Med 371:974-976

7. Reid IR (2015) Efficacy, effectiveness and side effects of medications used to prevent fractures. J Intern Med 277:690-706

8. Papapoulos S, Lippuner K, Roux C et al (2015) The effect of 8 or 5 years of denosumab treatment in postmenopausal women with osteoporosis: results from the FREEDOM Extension study. Osteoporos Int. doi:10.1007/s00198-015-3234-7

9. Reid IR, Miller PD, Brown JP et al (2010) Effects of denosumab on bone histomorphometry: the FREEDOM and STAND studies. J Bone Miner Res 25:2256-2265

10. Black DM, Reid IR, Cauley JA et al (2015) The effect of 6 versus 9 years of zoledronic acid treatment in osteoporosis: a randomized second extension to the HORIZON-pivotal fracture trial (PFT). J Bone Miner Res 30:934-944
11. Ferrari S, Adachi JD, Lippuner K et al (2015) Further reductions in nonvertebral fracture rate with long-term denosumab treatment in the FREEDOM open-label extension and influence of hip bone mineral density after 3 years. Osteoporos Int. doi:10.1007/s00198015-3179-x.

12. Gnant M, Pfeiler G, Dubsky PC et al (2015) Adjuvant denosumab in breast cancer (ABCSG-18): a multicentre, randomised, doubleblind, placebo-controlled trial. Lancet 386:433-443

13. Black DM, Delmas PD, Eastell R et al (2007) Once-yearly zoledronic acid for treatment of postmenopausal osteoporosis. N Engl J Med 356:1809-1822

14. Black DM, Reid IR, Cauley J et al (2011) The effect of 3 versus 6 years of zoledronic acid treatment in osteoporosis: a randomized extension to the HORIZON-Pivotal Fracture Trial (PFT). Bone 48:S91-S91

15. Lyles KW, Colon-Emeric CS, Magaziner JS et al (2007) Zoledronic acid and clinical fractures and mortality after hip fracture. N Engl J Med 357:1799-1809

16. Black DM, Kelly MP, Genant HK et al (2010) Bisphosphonates and fractures of the subtrochanteric or diaphyseal femur. N Engl J Med $362: 1761-1771$ 